\title{
Communication with patients from other cultures: the place of explanatory models
}

\author{
Kamaldeep Bhui \& Dinesh Bhugra
}

\begin{abstract}
We discuss the complicated nature of communication between people from different cultural groups, perhaps using a second language. We focus on the fact that mental health practitioners and service users often have in common neither their cultural backgrounds nor their explanatory models of illness. Communication even in a shared language can be less than optimal as words carry multiple meanings. Consequently, consultations that involve culturally grounded explanatory models of illness challenge the professional. We give examples showing that reconciling different explanatory models during the consultation is a core task for psychiatrists and mental health practitioners working in multicultural settings.
\end{abstract}

It is nearly two decades since Kleinman (1980) proposed the wider acceptance of the role of explanatory models in the assessment and management of mental disorders. The 'explanatory model' concept was intended to draw on social-anthropological approaches to understanding subjective experiences of distress and to apply them to psychiatric practice (Bhui \& Bhugra, 2002a). Pleas to recruit 'understanding' and 'empathy' into the clinical method have been with us since Jaspers' early writings on general psychopathology (Broome, 2002). The tension between identifying and understanding abnormalities of mental state persists into current psychiatric practice. Consultations are increasingly regarded, mainly by non-psychiatrists but also by some psychiatrists, as a technological enterprise. Checklists, clinical guidelines, clinical protocols, risk assessment tools, local implementation plans for the National Service Framework, governance requirements, appraisal and CPD portfolios, teaching portfolios and membership of learned institutions all include lists of activities, objectives and achievements. These documents regulate our practice by ensuring that minimum standards are met, and they demonstrate that our work includes more than sound clinical practice. None the less, less attention is now paid to the more human aspects of psychiatry, which rely on sound clinical practice and include 'quality in the clinical method', consultation dynamics, effective history-taking, understanding, empathy and building a therapeutic alliance taking account of transferential and countertransferential issues.

\section{Practising in a multicultural context}

Drenan \& Swarz (2002) remind us that psychiatric practice in multi-lingual settings involves various people acting as interpreters, which often leads to different conclusions about the significance of expressions of distress; for example, whether they indicate psychopathology or are culturally grounded and therefore not abnormal. It is often difficult for doctors to realise that family members' own explanation is one of many that patients hold in mind. Furthermore, Williams \& Healy (2001) point out that explanatory models are not fixed and stable representations but that they fluctuate and are recruited in a context-dependent manner. This perhaps persuades doctors that explanatory models are not important or influential. Certainly, when actual (observed) behaviour does not map onto reported behaviour (expressed in an explanatory model), this needs to be factored into clinical decision-making. Explanatory models represent the position from which patients may express distress; and they can govern how patients interpret a psychiatric explanation of their problems. Even if patients unconditionally accept the medical perspective, there is still scope for explanatory models to influence adherence to treatment, especially if family, community and some personal explanations are at variance with the medical model.

Kamaldeep Bhui is Professor of Cultural Psychiatry and Epidemiology at St Bartholomew's and The Royal London School of Medicine and Dentistry (Queen Mary and Westfied College, Mile End Road, London E1 4NS, UK. E-mail: k.s.bhui@qmul.ac.uk). He is interested in cross-cultural and epidemiological psychiatry, service development and explanatory models of illness. Dinesh Bhugra is Professor of Mental Health and Cultural Diversity and heads the Section of Cultural Psychiatry at the Institute of Psychiatry, London. His research interests include cultural factors in the aetiology and diagnosis of mental illness. 


\section{The process of exploring explanatory models}

The concept of explanatory models as it was originally formulated appears to have had little impact on psychiatric practice in general, even though there is now a greater emphasis on user views and satisfaction (Bhui \& Bhugra, 2002b). Patients' explanatory models are not fixed and are influenced by the circumstances of their symptoms, but they can influence a physician's assessments (Bhui et al, 2002; Bhui \& Bhugra, 2002a).

It is the process of exploring with the patient his or her identity and explanatory model that ensures improved understanding and informs the successful negotiation of different world views. This exploration does not require psychiatrists to enter into another culture as a participant observer, a prerequisite for any form of authentic ethnography. Nor does it require them to undergo in-depth psychoanalysis of their own world view. However, they do have to transfer models of mind and functioning from these disciplines into the therapeutic clinical setting. Personal psychotherapy is core to the training of psychiatrists and this may be an appropriate opportunity to begin explorations of cultural differences. It should be remembered, though, that psychotherapeutic theories and practice have their own cultural biases, which are often made manifest in a mismatch at a theoretical, technical or philosophical level (Bhugra \& Bhui, 1998, 2002).

\section{Cultural formulations}

Cultural formulations were introduced into DSMIV in an attempt to make diagnostic practice more culturally appropriate, relevant and representative. This marked a beginning of the exploration of values in diagnostic criteria, but cannot replace a thorough exploration of the values of patient and professional during the clinical process. The American Psychiatric Association (2002) recommends five elements in the cultural formulation (Box 1). The second of these relates to the patient's explanatory model of the illness, and explores cultural factors beyond race and ethnicity. However, in isolation from the other elements, awareness of explanatory models is unlikely to influence the quality of the consultation, the assessment or the management of the patient's distress.

Readers might like to explore what further information a cultural formulation might have yielded to influence the management of the young man in the following case history. (For conciseness, the full cultural formulation of Mr B's beliefs is not reproduced here.)
Case history 1

Mr B was an 18-year-old Bangladeshi man under investigation for unexplained physical symptoms by gastroenterological, orthopaedic and general medical services in a teaching hospital. His general practitioner knew that the young man was very distressed and was avoiding school, but could not reassure him. The specialists could find no organic illness. Mr B was seen by two senior psychiatrists (one English and one Indian: no Bangladeshi psychiatrist was then available), and their overall view was that his symptoms were psychosomatic but that his unwillingness to attend psychiatric appointments and failure to communicate with them made it unlikely that he would come to understand why he would not benefit from further physical investigations.

Mr B was eventually seen by another Indian psychiatrist, who found that Mr B spoke English well. Unlike the two previous psychiatrists, the third psychiatrist was able to explore Mr B's problems from the young man's own perspective. During the course of the assessment sessions $\mathrm{Mr} B$ revealed that he had first noticed 'stomach noises' in the mosque and had immediately attributed them to an abnormal mass in his stomach - stomach cancer or something equally 'bad'. He became unable to visit the mosque, which distressed both him and his father. Both were concerned that he could not remain active in worship within the local Muslim community, as this would have violated their religious practices. He also admitted that he had palpitations and sweats and became frightened when his stomach made the noises.

The psychiatrist and Mr B acknowledged the latter's belief in this growth in his stomach as both important and disabling, causing much concern to his family. However, the psychiatrist pointed out that there was no evidence of any physical abnormality and recommended an antidepressant, explaining that this fitted into his schema of what was causing Mr B's problems. It was thus explicitly acknowledged that Mr B might have been unfamiliar with this remedy. The young man's belief that there was a mass in his stomach resonated with the phenomenon of Tharan, which the psychiatrist had previously encountered among patients of Indian origin. Tharan is described

Box 1 The five elements of cultural formulation (American Psychiatric Association, 2002)

1 The cultural identity of the individual

2 Cultural explanations of the individual's illness

3 The influence of the patient's psychosocial environment and level of functioning within it

4 Cultural elements in the patient-professional relationship

5 The use of cultural assessment in deciding diagnosis and care 
as a mass in the stomach, for which the treatment is massage to remove the blockage.

This approach of negotiation without conflict, in which both the psychiatrist and the patient respectfully accepted alternative, perhaps to each unusual, explanatory models led $\mathrm{Mr} \mathrm{B}$ to accept antidepressant medication, and within 2 weeks he was much less anxious.

The psychiatrist was then able to instigate a series of cognitive-behavioural therapy (CBT) sessions. The CBT was targeted at enabling the young man to return to school and to the mosque, by encouraging response prevention and by cognitively challenging his morbid explanation for the stomach noises (but not challenging his cultural explanation of them). Although Mr B missed 4 of the 16 CBT sessions, he always left a message and followed the behavioural homework tasks. He always came to the clinic with his friends, who waited outside for him. He resumed weekly attendance at the mosque and returned to school, and the stomach noises settled. His explanation of what had led to recovery included regular stomach massage by his father to remove the mass. He thought that the antidepressant medication had helped him, but had no idea whether the CBT had also been useful. Tolerance of his failure to attend all of the CBT sessions, acceptance of his explanations and the psychiatrist's not insisting on taking the credit for recovery enabled $\mathrm{Mr} \mathrm{B}$ and his father to find a solution that helped relieve their personal, family and social distress.

This case shows how the patient's explanatory models of illness can be creatively used in consultations and need not compete with the psychiatrist's model. The psychiatrist does not have to be from the same cultural background to achieve a therapeutic alliance. It also illustrates that evidence-based interventions can be delivered despite differing explanatory models.

\section{Communication, culture and therapeutic relationships}

What is therapeutic in a clinical situation? What makes up a therapeutic relationship? The microskills necessary to maximise the therapeutic alliance and relationship are the cornerstone of sound clinical practice and are exercised during the consultation, when patient meets psychiatrist. Yet less is known about these key aspects of clinical practice than about most pharmacological interventions (McGuire et al, 2001). The psychiatrist's primary aim is to gather authentic information, make judgements about psychopathology and then understand how best to make use of this information to achieve the therapeutic goals brought to the consultation by the patients, as well as introduce the psychiatrist's own objectives. This is far from a technical process. So when this complex and valueladen task includes patients from a different cultural background, there is even more potential for miscommunications to enter the discourse.

\section{The problem with words}

Acts of speech are often taken for granted as straightforward communications of particular realities. In fact, in a psychiatric setting they are a discourse about individual/private and societal/ public issues expressed through languages of distress. The multiple meanings carried by each word, when understood as being reflexively shaped by other words in the same sentence and by the biography of the individual, are also strongly connected with cultural meanings, symbols (social and political), and historical and contemporary narratives. The manifest and latent meanings attached to any word, even in the same language, may differ between patient and clinician. Said, a Christian Palestinian living in exile in New York, writes about communication:

'The language in which we are speaking is his before it is mine. How different are the words home, Christ, ale, master, on his lips and on mine! I cannot speak or write these words without unrest of spirit. His language, so familiar and so foreign, will always before me be an acquired speech' (Said, 1983: p. 48).

A similar position was taken by Lacan (see Nobus, 2000: p. 71). Thus, for both the child and the adult language is most inadequate for the communication of needs, which are more easily expressed symbolically. Even for people who share the same mother tongue, language can lead to miscommunications in symbolic terms. How much more difficult must it be to communicate deep needs in a second language. In a study of Somali refugees, six translators spent at least 40 hours ensuring that the translated instruments had face and content validity. Clinicians are rarely able to take into account the many factors that influence communication and assessment of emotions across cultures.

\section{Recognising emotions}

Despite international research (Shioiri et al, 1999) showing that a finite number of emotions are recognised in all societies and cultures (surprise, disgust, fear, anger, contempt, happiness and sadness), it is known that the accurate recognition of these emotional states varies with culture and observer (Shioiri et al, 1999; Elfenbein \& Ambady, 2002,2003 ) and becomes more precise the greater the exposure to the culture of the person being assessed. It may be that when psychiatrists assess 
emotional states across cultures, and even across socio-economic groups, the emotional content is not fully appreciated. Such fine-grain omissions might account for some of the dissatisfaction felt by ethnic minorities and perhaps for some of the perceived lower involvement of professionals with patients from minority groups. For example, general practitioners in the south London borough of Camberwell reported that they felt less involved in the care of their Black patients, but on examination of the records it was found that they had just as much instrumental involvement with them as they had with their White patients (Bindman et al, 1997).

\section{Empathy}

Understanding barriers to empathy across cultures sheds light on why psychiatric assessment across cultures is difficult or imprecise. Exploring the explanatory models held by service users may allow a broader exploration of the affects and emotions associated with their own understanding of their problems. It is known that speaking in a second language can have a defensive function (Tesone, 1996); perhaps speaking through some one else's explanatory model serves a similar function, making the other's model seem unreal or unintelligible.

\footnotetext{
Case history 2

A 24-year-old Bangladeshi man sustained a head injury in a road traffic accident. He presented with impulsiveness and poor judgement; frontal lobe damage was evident on magnetic resonance imaging (MRI). He believed the accident and its consequences to be an act of God. His psychiatrist believed that the young man's behaviour and poor functioning resulted from a head injury sustained during the accident. The two could not agree, and the man's family were invited to discuss the reasons for his condition. They also supported the view that God is superordinate and responsible for everything, including the abnormal MRI scan and the young man's symptoms. The mental health team feared that this belief in God would impair the man's ability to adhere to treatment, and were troubled that the family did not accept the medical diagnosis and explanation, which was based on the scan evidence.
}

This case history illustrates quite distinct but competing explanations for behavioural change. The psychiatrist and mental health team are used to holding a single explanation for events, one which is evidence based and grounded on the scientific foundations of psychiatry. The young man and his family, who have strong religious beliefs, understand the implications of the abnormal scan, but are preoccupied with the broader meaning of the event in terms of the forces they believe to govern the world. Interestingly, they are able to hold competing beliefs without feeling any inner conflict, whereas the mental health team view the family's powerfully held unscientific religious explanations with suspicion.

Differences in religious world view, experiences of health services, expectations of recovery, and lay referral systems in the folk and popular sector may all contribute to the divergence of explanatory models between professionals and patients.

\section{When compromise is difficult}

Even if explanatory models differ markedly between professional and patient, it should be possible to reach a compromise quite quickly. By avoiding antagonistic and conflictual styles of consultation and permitting the patient to hold on to their own explanations, the professional should be able to persuade patients that is it OK to 'agree to differ'. This means that members of mental health teams must respect their patients' views just as much as they expect patients to respect theirs.

Sometimes, an impasse is reached where neither side can agree to accept and work with the other's viewpoint. This situation resembles that in which a patient in general medical practice refuses to adopt the sick role, which is quite common. The manner in which this dispute is resolved will be coloured by the values and attitudes of the doctor towards both patients and ill health. Furthermore, differences in education and social class influence ability to negotiate barriers. In cross-cultural consultations it is common for specifically cultural factors also to influence the management of such an impasse (Bhugra \& Bhui, 2002). Doctors often feel that the patient's view is exotic, unscientific and, more specifically, embedded in a cultural world view that they do not understand. Such situations often end up as case presentations and spark grand-round debates about how culture influences psychopathology.

Among mental health professionals there is often a fundamental lack of understanding and valuing of foreign or non-psychiatric perspectives. Added to this, the task of reaching consensus with patients is itself loaded with the potential to perpetuate or exacerbate this lack of understanding if mutually agreed rules of discussion and negotiation to build a therapeutic alliance are not prominent. Leff (1988) has described how traditional healers tend to consult in public, with consensus and sanction from the wider community. More specifically, he notes that they skilfully negotiate difference of opinion and attitude using their knowledge of the culture in which they are embedded. Negotiation of differences in world view may be a useful core skill for psychiatrists working in multicultural settings. 
Exploring cultural identity with patients allows them to express their culturally determined views, which will have been shaped both by the subculture and the dominant society in which they live. Such an approach is as relevant for White majorities and their subcultural groups as it is for minority ethnic communities.

\section{References}

American Psychiatric Association (2002) Cultural Assessment in Clinical Psychiatry. Washington, DC: American Psychiatric Publishing.

Bhugra, D. \& Bhui, K. (1998) Psychotherapy for ethnic minorities. British Journal of Psychotherapy, 14, 310-326.

Bhugra, D. \& Bhui, K. (2002) Is the Oedipal complex universal? Problems for sexual and relationship psychotherapy across cultures. Sexual and Relationship Therapy, 17, 69-86.

Bhui, K. \& Bhugra, D. (2002a) Explanatory models for mental distress: implications for clinical practice and research (editorial). British Journal of Psychiatry, 181, 6-7.

Bhui, K. \& Bhugra, D. (2002b) Mental illness in Black and Asian ethnic minorities: pathways to care and outcomes. Advances in Psychiatric Treatment, 8, 26-33.

Bhui, K., Bhugra, D. \& Goldberg, D. (2002) Causal explanations of distress and general practitioners' assessments of common mental disorder among Punjab and English attendees. Social Psychiatry and Psychiatric Epidemiology, 37, 38-45.

Bindman, J., Johnson, S., Wright, S., et al (1997) Integration between primary and secondary services in the care of the severely mentally ill: patients' and general practitioners' views. British Journal of Psychiatry, 171, 169-174.

Broome, M. (2002) Explanatory models in psychiatry. British Journal of Psychiatry, 181, 351-352.

Drenan, G. \& Swarz, L. (2002) The paradoxical use of interpreting in psychiatry. Social Science and Medicine, 54, 1853-1866.

Elfenbein, H. A. \& Ambady, N. (2002) On the universality and cultural specificity of emotion recognition: a metaanalysis. Psychological Bulletin, 128, 203-235.

Elfenbein, H. A. \& Ambady, N. (2003) When familiarity breeds accuracy: cultural exposure and facial emotion recognition. Journal of Personality and Social Psychology, 85, 276-290.

Kleinman, A. (1980) Patients and Healers in the Context of Culture. Thesis. Berkley, CA: University of California Press.

Leff, J. (1988) Psychiatry Around the Globe: A Transcultural View (2nd edn). London: Gaskell.

McGuire, R., McCabe, R. \& Priebe, S. (2001) Theoretical frameworks for understanding and investigating the therapeutic relationship in psychiatry. Social Psychiatry and Psychiatric Epidemiology, 36, 557-564.

Nobus, D. (2000) Jacques Lacan and the Freudian Practice of Psychoanalysis. London. Routledge.

Said, E. (1983) The World, and the Text and the Critic. Cambridge: MA. Harvard University Press.

Shioiri, T., Someya, T., Helmeste, D., et al (1999) Misinterpretation of facial expression: a cross-cultural study. Psychiatry and Clinical Neuroscience, 53, 45-50.

Tesone, J. E. (1996) Multi-lingualism, word-presentations, thing-presentations and psychic reality. International Journal of Psychoanalysis, 77, 871-881.

Williams, B. \& Healy, D. (2001) Perceptions of illness causation among new referrals to a community mental health team: explanatory model or exploratory map? Social Science and Medicine, 53, 465-476.

\section{MCQs}

1 As regards identification and meaning of explanatory models:

a only anthropologists can elicit explanatory models

$\mathrm{b}$ participant observation is essential for identifying the explanatory models of patients from other cultures

c explanatory models are cognitive representations of insight-related beliefs

d explanatory models are delusional, but culturally determined delusions

e talking to the patient's family and peer group can help unravel the cultural origins of their explanatory models.

2 As regards explanatory models in the clinical consultation:

a explanatory models can be treated with neuroleptics

b explanatory models are stable representations of a patient's world view about their problems

c negotiation of different explanatory models is key to improved communication

d a multi-lingual interpreter should be able to relay a complete picture of a patient's world view

e patients who replace their own explanatory model with that of the psychiatrist are likely to be totally compliant with the psychiatrist's recommendations.

3 A patient's explanatory models can be ascertained by asking:

a about the name given to symptoms

b why symptoms have occurred at this time

c why symptoms will respond to a specific therapy

$\mathrm{d}$ why the patient is frightened of these symptoms

e what treatment is required.

4 The following factors commonly influence the basic features of explanatory models:

a age

$b$ gender

c history of good physical health

d educational status

e time-point and context of assessment.

5 Cultural formulation includes:

a the therapist's view of the patient's cultural identity

b cultural explanations of distress

c the influence of the psychosocial environment

d cultural elements in the patient-therapist relationship

e noting the patient's culture in reaching a diagnosis.

\begin{tabular}{|c|c|c|c|c|}
\hline \multicolumn{5}{|c|}{ MCQ answers } \\
\hline 1 & 2 & 3 & 4 & 5 \\
\hline a $F$ & a $F$ & a $\mathrm{T}$ & a $\mathrm{T}$ & a $F$ \\
\hline b F & b F & $\mathrm{b} \mathrm{T}$ & $\mathrm{b} \mathrm{T}$ & $\mathrm{b} \mathrm{T}$ \\
\hline c F & c $\mathrm{T}$ & c $\mathrm{T}$ & c F & c $\mathrm{T}$ \\
\hline d F & d F & $\mathrm{d} \mathrm{T}$ & $\mathrm{d} \mathrm{T}$ & $\mathrm{d} \mathrm{T}$ \\
\hline e $\mathrm{T}$ & e $F$ & e $\mathrm{T}$ & e $\mathrm{T}$ & e $\mathrm{T}$ \\
\hline
\end{tabular}

